\title{
Effects of post-interventional antiplatelet therapy on angiographic vasospasm, delayed cerebral ischemia, and clinical outcome after aneurysmal subarachnoid hemorrhage: a single-center experience
}

\author{
Claudia Ditz ${ }^{1}$ (D) Björn Machner ${ }^{2} \cdot$ Hannes Schacht ${ }^{3} \cdot$ Alexander Neumann $^{3} \cdot$ Peter Schramm $^{3} \cdot$ Volker M. Tronnier $^{1}$. \\ Jan Küchler ${ }^{1}$
}

Received: 1 October 2020 / Revised: 22 December 2020 / Accepted: 8 January 2021 / Published online: 25 January 2021

(C) The Author(s) 2021

\begin{abstract}
Platelet activation has been postulated to be involved in the pathogenesis of delayed cerebral ischemia (DCI) and cerebral vasospasm (CVS) after aneurysmal subarachnoid hemorrhage (aSAH). The aim of this study was to investigate potentially beneficial effects of antiplatelet therapy (APT) on angiographic CVS, DCI-related infarction and functional outcome in endovascularly treated aSAH patients. Retrospective single-center analysis of aSAH patients treated by endovascular aneurysm obliteration. Based on the post-interventional medical regime, patients were assigned to either an APT group or a control group not receiving APT. A subgroup analysis separately investigated those APT patients with aspirin monotherapy (MAPT) and those receiving dual treatment (aspirin plus clopidogrel, DAPT). Clinical and radiological characteristics were compared between groups. Possible predictors for angiographic CVS, DCI-related infarction, and an unfavorable functional outcome (modified Rankin scale $\geq 3$ ) were analyzed. Of 160 patients, $85(53 \%)$ had received APT $(n=29$ MAPT, $n=56$ DAPT). APT was independently associated with a lower incidence of an unfavorable functional outcome (OR 0.40 [0.19-0.87], $P=0.021)$ after 3 months. APT did not reduce the incidence of angiographic CVS or DCI-related infarction. The pattern of angiographic CVS or DCI-related infarction as well as the rate of intracranial hemorrhage did not differ between groups. However, the lesion volume of DCI-related infarctions was significantly reduced in the DAPT subgroup $(P=0.011)$. Post-interventional APT in endovascularly treated aSAH patients is associated with better functional outcome at 3 months. The beneficial effect of APT might be mediated by reduction of the size of DCI-related infarctions.
\end{abstract}

Keywords Antiplatelet therapy · Cerebral vasospasm - Delayed cerebral ischemia · Endovascular treatment · Aneurysmal subarachnoid hemorrhage

Claudia Ditz

claudia.ditz@uksh.de

1 Department of Neurosurgery, Universitätsklinikum Schleswig-Holstein, Campus Lübeck, Ratzeburger Allee 160, 23538 Lübeck, Germany

2 Department of Neurology, Universitätsklinikum Schleswig-Holstein, Campus Lübeck, Lübeck, Germany

3 Department of Neuroradiology, Universitätsklinikum Schleswig-Holstein, Campus Lübeck, Lübeck, Germany

\section{Introduction}

Delayed cerebral ischemia (DCI) is a severe complication after aneurysmal subarachnoid hemorrhage (aSAH) and represents the most important predictor of mortality and morbidity in patients who survive the acute bleeding event $[18,37]$. DCI usually occurs between 4 and 10 days after the initial hemorrhage. It is clinically defined by the development of new neurological deficits or a sudden decrease of consciousness [50] and may lead to secondary cerebral infarction and permanent disability $[35,47,50]$. The pathophysiological mechanisms involved in DCI are complex and still not completely understood. Cerebral vasospasm (CVS), characterized by angiographic vasoconstriction of arterial blood vessels, plays an 
important role in the development of DCI $[10,17]$. However, current research proposes a multifactorial nature of DCI beyond the traditionally emphasized CVS. Several mechanisms have been postulated to be involved including cortical spreading depolarization, inflammatory response, activation of the coagulation cascade, and microthrombosis [3, 28, 38, 49]. In particular, increased platelet activation after SAH is considered to contribute to the development of DCI. Endothelial damage from aneurysm rupture leads to immediate platelet activation and aggregation with consecutive release of cytokines and vasoactive substances from within the platelet granules. These processes do not only cause vasoconstriction and the formation of microthrombi but also contribute to the activation of inflammation [48]. Accordingly, an increased platelet activity after aSAH with consecutive release of thromboxane A2 was found to be associated with DCI and the occurrence of angiographic CVS [25, 26, 31]. Moreover, the antiplatelet activity of the endothelium is reduced after aSAH resulting in an increased platelet adhesion and formation of microthrombi [30]. Previous studies suggest that microthrombi/-emboli play an essential role in the development of DCI-related infarction [34, 41, 42, 48].

Several clinical studies have therefore evaluated possible preventive effects of different antiplatelet agents such as aspirin on the development of CVS, DCI, and functional outcome after aSAH [15, 20, 24, 45, 46]. However, the results of these studies are contradictory and quite heterogeneous with regard to the investigated antiplatelets, the start and duration of treatment, and the investigated endpoints. Moreover, most of the older studies in the pre-ISAT era primary report on surgically treated patients, whereas endovascularly treated patients are underrepresented. Accordingly, appropriate treatment recommendations regarding the use of antiplatelets in aSAH patients are still missing. However, in view of the current developments toward an increasing role of endovascular aneurysm treatments, appropriate risk-benefit assessments and recommendations regarding APT in patients with acute SAH are needed. A growing number of aSAH patients require postinterventional APT with aspirin or even dual antiplatelet therapy (DAPT, aspirin plus clopidogrel), due to stent or flow diverter-assisted aneurysm treatments. Hence, recent series focused on the effects of post-interventional APT among endovascularly treated aSAH patients $[4,11,29,51]$.

The purpose of this study was to retrospectively evaluate the effects of APT (aspirin with/without additional clopidogrel) on functional outcome, the occurrence of angiographic CVS, and the prevention of DCI-related infarction in a collective of endovascularly treated aSAH patients. In particular, we compared characteristic patterns of angiographic CVS and DCI-related infarction between the groups and assessed the risk of hemorrhagic complications. We aimed to provide additional guidance to the ongoing debate on the value of APT in aSAH patients.

\section{Patients and methods}

This study was approved by the Institutional Review Board (20-257). For this type of retrospective study, informed consent is not required. We screened all consecutive patients who were admitted to our institution between January 2011 and December 2019 for the following inclusion criteria: (i) SAH secondary to the rupture of an intracranial aneurysm, (ii) endovascular aneurysm treatment. The exclusion criteria were as follows: surgical treatment of the aneurysm, no treatment of the aneurysm due to devastating aSAH, early death $(<7$ days after admission), aSAH associated with arteriovenous malformation or mycotic aneurysm, coiling of the aneurysm with the need for secondary stenting later within the acute clinical course, stent-assisted coiling without subsequent administration of antiplatelets, evidence of CVS on admission angiography, and the use of heparin, e.g., due to deep-vein thrombosis or acute coronary syndrome.

General demographic information and clinical and radiological data were collected by chart review. Patients receiving post-interventional antiplatelet medication (aspirin \pm clopidogrel) were assigned to the "APT group," while the "control group" consisted of patients without antiplatelet medication after endovascular aneurysm repair. A subgroup analysis was performed within the "APT" group investigating patients with either mono- (aspirin) or dual (aspirin plus clopidogrel) APT.

Hemorrhagic intracranial complications were assessed on regular follow-up non-enhanced computed tomography (NCT) and categorized into minor and major bleeding events, whereas major events were defined as new intracranial hemorrhage that led to a clinical deterioration and/or necessitated additional surgery.

Functional outcome was assessed at 3 months after discharge using the modified Ranking Scale (mRS) and dichotomized into favorable $(\mathrm{mRS} \leq 2)$ and unfavorable outcome $(\mathrm{mRS} \geq 3)$.

\section{Definition and analysis of $\mathrm{DCl}, \mathrm{DCl}$-related infarction, and angiographic CVS}

DCI was defined clinically as the development of new focal neurological deficits and/or a decrease in Glasgow Come Scale $\geq 2$ points, lasting for more than $1 \mathrm{~h}$ (delayed ischemic neurologic deficit, DIND), and/or by imaging criteria as new DCI-related infarction on follow-up NCT or magnetic resonance imaging (MRI) [50]. In analgosedated patients, who evade neurological assessments, the definition of DCI was expanded to include events of functional deterioration such as a refractory decrease in $\mathrm{PBrO}_{2}$ below $15 \mathrm{mmHg}$ or DCIrelated hypoperfusion on PCT as defined below. Events of clinical or functional DCI were documented and compared between the groups. However, as our study cohort consisted 
of awake and unconscious/analgosedated patients, data on clinical or functional DCI were only partially available and difficult to compare. Thus, only the occurrence of DCIrelated infarction, as defined by radiological criteria, was analyzed as an independent endpoint in multivariable analysis as this outcome parameter was assessable in all patients and has the greatest impact on clinical outcome.

DCI-related infarction was defined as new hypodense lesions on follow-up CT or as new diffusion-restricted areas on MRI not attributable to other causes. Pre-existing infarctions and infarctions related to the primary brain damage, cerebral herniation, or the initial aneurysm treatment were excluded. Hypodensities resulting from intracerebral hematoma or surgical interventions were not regarded as infarction.

DCI-related infarctions were grouped into the following categories: single or multiple vascular territories, cortical or deep location, and unilateral or bilateral occurrence [33].

The NCT scans of all patients were inspected by two experienced neuroradiologists, and DCI-related infarctions were manually delineated on the individual patient image using the MRIcron software (http://www.mricro.com/mricron) [36]. Next, the delineated lesion of every patient was normalized into the standardized MNI (Montreal Neurological Institute) space using the clinical toolbox in SPM (Statistical Parametric Mapping, version 12, http://www.fil.ion.ucl.ac.uk/spm; Wellcome Trust Centre for Neuroimaging, London, UK), implemented in Matlab® 2018B (MathWorks ${ }^{\circledR}$, Natick, MA). Using MRIcron, lesion overlap images were created for each group to illustrate the location, distribution, and extent of DCI-related infarctions. Furthermore, the individual lesion volume was derived and used for further statistical analyses.

Angiographic CVS was defined as the occurrence of new arterial vessel narrowing on follow-up digital subtraction angiography (DSA) (compared with the vessel diameter in the initial DSA performed on admission). Severity and distribution of angiographic CVS were assessed for each vessel by the neuroradiologist who performed the intervention and verified retrospectively by a second experienced interventional neuroradiologist. The number of affected vessels per patient and the affected vessel segments (proximal/ distal) in each DSA as well as the number and location of intraarterial nimodipine (IAN) treatments and/or balloon angioplasty were collected. Proximal angiographic CVS was defined as CVS in the internal carotid artery, the vertebral or basilar artery, and within the first segments of the anterior, middle, and posterior cerebral artery. The degree of angiographic CVS was rated as "mild" when narrowing of the arterial diameter $<29 \%$, "moderate" when 30 to $49 \%$, and "severe" when $\geq 50 \%$, each compared with the vessel diameter on the initial angiography.

\section{Clinical management of aSAH patients}

All patients with aSAH were managed in the neurointensive care unit under the care of the neurosurgery service and in accordance with current recommendations [9]. Four-vessel DSA was performed in all patients in order to evaluate the presence of a ruptured aneurysm. In case of acute hydrocephalus or severe IVH, cerebrospinal fluid (CSF) drain was placed prior to the endovascular intervention. The choice of endovascular treatment was made individually upon interdisciplinary assessment based on the aneurysm location and configuration as well as on demographic and clinical factors. Endovascular aneurysm treatment was performed within $48 \mathrm{~h}$ after admission.

The decision about the use of APT after coil embolization was made by the treating neuroradiologist depending on the expected risk of thromboembolic complications (e.g., wide aneurysm base, protrusion of a coil in the vessel lumen). If indicated after coil embolization, aspirin was administrated in a daily dose of $100 \mathrm{mg}$ for approximately 4 to 12 weeks. If remodeling stents or flow diverters were used for acute treatment, intravenous tirofiban infusion was started with a basal-bolus regimen of $0.4 \mu \mathrm{g} / \mathrm{kg} / \mathrm{min}$ for $30 \mathrm{~min}$ followed by a $0.10 \mu \mathrm{g} / \mathrm{kg} / \mathrm{min}$ maintenance infusion until post-interventional complications were ruled out by followup NCT scans. If necessary, tirofiban infusion was paused to allow the placement of an external ventricular drain (EVD) or invasive neuromonitoring probes. Subsequently, loading with $300 \mathrm{mg}$ aspirin and $450 \mathrm{mg}$ clopidogrel was performed and followed by a daily maintenance dose of $100 \mathrm{mg}$ aspirin and $75 \mathrm{mg}$ clopidogrel. DAPT was continued for at least 6 weeks while aspirin use was continued in most cases. Platelet function tests were not routinely performed in all included patients.

After aneurysm treatment, the patients were kept normovolemic with a mean arterial pressure (MAP) above $80 \mathrm{mmHg}$. Standard antithrombotic therapy included subcutaneous low-dose heparin starting from day 1 after aneurysm treatment. All patients received nimodipine, administered orally at a dose of $60 \mathrm{mg}$ every $4 \mathrm{~h}$ or intravenously with $1-$ $2 \mathrm{mg}$ of nimodipine per hour.

All patients were monitored for DCI and CVS with serial neurologic examinations and daily transcranial Doppler (TCD) measurements of flow velocities (FVs) in the large cerebral arteries.

Routine monitoring for DCI and CVS included serial neurologic examinations in awake patients and daily transcranial Doppler (TCD) measurements. Analgosedated patients such as patients with poor-grade sSAH (World Federation of Neurosurgical Societies (WFNS) grade 4-5) received an invasive multimodal neuromonitoring for intracranial pressure (ICP), cerebral perfusion pressure (CPP), and brain tissue oxygenation $\left(\mathrm{PBrO}_{2}\right)$. The neuromonitoring probes were placed in the frontal watershed area of the hemisphere ipsilateral to the ruptured aneurysm and in most cases additionally in the contralateral hemisphere. An increase of TCD mean FV of $150 \mathrm{~cm} / \mathrm{s}$ or an increase of FV by $50 \mathrm{~cm} / \mathrm{s}$ over $24 \mathrm{~h}$, as well as a refractory decrease in $\mathrm{PBrO}_{2}$ below $15 \mathrm{mmHg}$, was considered as indications of CVS or DCI. 
Follow-up imaging included an early post-interventional NCT (within $24 \mathrm{~h}$ after aneurysm treatment) in all patients. Further follow-up NCT scans were performed during the hospital stay depending on the clinical indication. Additional perfusion CT (PCT) examinations were performed in the event of clinical DCI (awake patients) or in case of functional suspicion of DCI or CVS. In analgosedated patients, PCTs were also performed routinely every 3 to 4 days according to our internal protocol [12]. Perfusion maps were qualitatively/ semi-quantitatively evaluated via visual inspection by two board-certified neuroradiologists to detect new DCI-related perfusion deficits, defined as areas of prolonged TTD not related to other causes. The TTD parameter map was chosen since it was formerly described to provide the best image quality and the highest contrast for delineating ischemic areas [13].

In case of clinical or functional DCI, hyperdynamic therapy was initiated as recommended [9] by raising the systolic arterial blood pressure above $180 \mathrm{mmHg}$ by the use of crystalloids and/or vasopressors. If the conservative treatment failed to improve the patients' clinical or functional condition, DSA was performed to confirm angiographic CVS and to initiate ERT upon interdisciplinary decision.

\section{Statistical analysis}

Data analysis was performed using the software IBM® SPSS® 25 (IBM; Armonk, NY, USA). Continuous data are presented as medians and interquartile ranges (IQRs) while categorical data are presented by counts and percentages. Categorical variables were analyzed using the chi-square test, or, if applicable, with the Fisher exact test. Continuous variables were analyzed using the Mann-Whitney $U$ test for nonnormally distributed data. A $P$ value $\leq 0.05$ was considered statistically significant.

We used a multivariable logistic regression with backward stepwise selection to analyze factors that might be associated with the occurrence of angiographic CVS, DCI-related infarction, and functional outcome. All factors that had $P$ values $<$ 0.05 in a previous univariable testing were included. Variables with $P$ values $<0.05$ were accepted as independent predictors. Odds ratios and associated $95 \%$ confidence intervals were reported for both the univariable and multivariable analyses.

\section{Results}

Between January 2011 and December 2019, a total of 307 patients with aSAH were treated at our institution and 282 patients received aneurysm obliteration. Overall, 93 patients were treated surgically (33\%), while 189 patients received endovascular aneurysm treatment (67\%). Middle cerebral artery aneurysms $(n=70)$ were predominantly secured by surgical clipping $(n=46,66 \%)$. After excluding 29 patients due to the above-mentioned exclusion criteria, 160 patients with aSAH and endovascular aneurysm treatment were included in the final analysis. Eighty-five patients $(53 \%)$ received APT (APT group), 1/3 of them had aspirin monotherapy $(n=29 / 85)$ and $2 / 3$ a DAPT consisting of aspirin and clopidogrel $(n=56 / 85)$. The remaining 75 patients $(47 \%)$ received no APT and constituted the control group.

Post-interventional platelet function test was performed in $35 / 85$ APT patients ( $41 \%$ ), all of whom received DAPT due to stent-assisted coiling. The majority of patients $(29 / 35,83 \%)$ showed a sufficient treatment response to both aspirin and clopidogrel, while 6 patients $(17 \%)$ responded insufficiently to APT (non-responsiveness to (i) both aspirin and clopidogrel $n=3$, (ii) aspirin only $n=1$, (iii) clopidogrel only $n=2$ ). At our institution, APT responder status was not assessed in case of post-interventional aspirin monotherapy.

The clinical characteristics of either group are shown in Table 1. There were no significant differences between the groups with regard to age, sex, WFNS and Fisher grade, acute hydrocephalus, aneurysm localization, or laterality. CVS drainage was required in $76 \%$ of the control group and in $64 \%$ of the APT group. EVD was placed in the majority of cases in both groups (74\% in the control group vs. $63 \%$ in the APT group), and most patients received the CVS drain prior to the endovascular aneurysm treatment $(65 \%$ in the control group vs. $59 \%$ in the APT group).

As expected, there was a statistically significant difference with regard to the modality of endovascular aneurysm treatment $(P<0.001)$ between the groups.

Treatment-related complications occurred in a similar percentage of patients in either group (13\% in the control group vs. $14 \%$ in the APT group), most of which were thromboembolic infarction and peri-interventional bleeding complications.

Clinical complications such as decompressive craniectomy or the need for ventricular peritoneal (VP) shunting as well as the inhospital mortality (11\% in the control group vs. $6 \%$ in the APT group, $P=0.386$ ) were similar between the groups. There was no significant difference regarding the incidence of any intracranial bleeding event (7\% in the control group vs. $6 \%$ in the APT group, $P=0.114)$. However, while minor bleeding events occurred more often in the control group (7\% vs. $2 \%$ ), major bleeding events were only observed in APT patients $(n=3,4 \%)$. One patient developed space-occupying epi- and subdural hematoma after decompressive craniectomy, while two patients developed large intracerebral hematoma after EVD or VP shunt placement, respectively. One patient died during hospitalization, and two patients had unfavorable outcome after 3 months.

Eighty-six patients (54\%) were awake and neurologically assessable during the clinical course. In these patients, DIND occurred in 39 cases (control group 17/36, 47\%; APT group $22 / 54,41 \% ; P=0.665)$. On the other hand, 74 patients $(46 \%)$ of the study cohort (control group $n=42,56 \%$; APT group 
Table 1 Clinical characteristics of the study cohort

\begin{tabular}{|c|c|c|c|}
\hline Parameter & Control group, $n=75$ & APT group, $n=85$ & $P$ value \\
\hline Age in years, median (IQR) & $55(47-65)$ & $54(48-65)$ & 0.801 \\
\hline Female sex, $n(\%)$ & $51(68)$ & $64(75)$ & 0.379 \\
\hline \multicolumn{4}{|l|}{ WFNS grade, $n(\%)$} \\
\hline I-III & $46(61)$ & $60(71)$ & \multirow[t]{2}{*}{0.243} \\
\hline IV-V & $29(39)$ & $25(29)$ & \\
\hline \multicolumn{4}{|l|}{ Fisher scale, $n(\%)$} \\
\hline $0-2$ & $9(12)$ & $17(20)$ & \multirow[t]{2}{*}{0.201} \\
\hline $3-4$ & $66(88)$ & $68(80)$ & \\
\hline Intraventricular hemorrhage, $n(\%)$ & $31(41)$ & $34(40)$ & 0.496 \\
\hline Intracerebral hemorrhage, $n(\%)$ & $14(19)$ & $9(11)$ & 0.178 \\
\hline Acute hydrocephalus requiring CSF drain, $n(\%)$ & $57(76)$ & $54(64)$ & 0.171 \\
\hline \multicolumn{4}{|l|}{ Aneurysm location, $n(\%)$} \\
\hline ICA & $11(15)$ & $17(20)$ & \multirow[t]{5}{*}{0.209} \\
\hline PComA & $9(12)$ & $3(4)$ & \\
\hline AComA and ACA & $30(40)$ & $29(34)$ & \\
\hline MCA & $7(9)$ & $12(14)$ & \\
\hline Posterior circulation & $18(24)$ & $24(28)$ & \\
\hline \multicolumn{4}{|l|}{ Aneurysm laterality, $n(\%)^{\mathrm{a}}$} \\
\hline Right side & $22(29)$ & $23(27)$ & \multirow[t]{2}{*}{0.406} \\
\hline Left side & $19(25)$ & $30(35)$ & \\
\hline \multicolumn{4}{|l|}{ Endovascular aneurysm treatment modality, $n(\%)$} \\
\hline Coil embolization & $75(100)$ & $29(34)$ & \multirow[t]{4}{*}{$<0.001$} \\
\hline WEB Device & $0(0)$ & $2(2)$ & \\
\hline Stent-assisted coil embolization & $0(0)$ & $51(60)$ & \\
\hline Flow diverter & $0(0)$ & $3(4)$ & \\
\hline Peri-interventional complications, $n(\%)$ & $10(13)$ & $12(14)$ & \multirow[t]{5}{*}{0.536} \\
\hline Thromboembolic infarction & $4 / 10(40)$ & $5 / 12(42)$ & \\
\hline Bleeding complication & $6 / 10(60)$ & $3 / 12(25)$ & \\
\hline Vascular dissection & $0 / 10(0)$ & $1 / 12(8)$ & \\
\hline Intraprocedural coil dislocation & $0 / 10(0)$ & $3 / 12(25)$ & \\
\hline Decompressive craniectomy, $n(\%)$ & $7(9)$ & $4(5)$ & 0.350 \\
\hline VP shunt, $n(\%)$ & $16(21)$ & $20(24)$ & 0.850 \\
\hline Length of stay (days), median (IQR) & $20(16-24)$ & $20(17-25)$ & 0.853 \\
\hline In-hospital mortality, $n(\%)$ & $8(11)$ & $5(6)$ & 0.386 \\
\hline Intracranial bleeding events, $n(\%)$ & $5(7)$ & $5(6)$ & 0.114 \\
\hline Minor & $5(7)$ & $2(2)$ & \\
\hline Major & $0(0)$ & $3(4)$ & \\
\hline \multicolumn{4}{|l|}{ Clinical or functional signs of DCI or CVS, $n(\%)$} \\
\hline Significant increase of FV in TCD & $25(33)$ & $30(35)$ & 0.868 \\
\hline DIND in awake patients & $17 / 36(47)$ & $22 / 54(41)$ & 0.665 \\
\hline \multicolumn{4}{|l|}{ Functional deterioration } \\
\hline $\mathrm{PBrO}_{2}<15 \mathrm{mmHg}$ in analgosedated patients & $19 / 34(56)$ & $10 / 27(37)$ & 0.198 \\
\hline DCI-related hypoperfusion in PCT & $34 / 53(64)$ & $18 / 53(34)$ & 0.003 \\
\hline DCI-related infarction, $n(\%)$ & $20(27)$ & $21(25)$ & 0.857 \\
\hline \multicolumn{4}{|l|}{ Vascular territories of DCI-related infarction, $n(\%)$} \\
\hline Single vascular territory & $10 / 20(50)$ & $10 / 21(48)$ & \multirow[t]{2}{*}{0.563} \\
\hline Multiple vascular territories & $10 / 20(50)$ & $11 / 21(52)$ & \\
\hline Location of DCI-related infarction, $n(\%)$ & & & \\
\hline Cortical & $14 / 20(70)$ & $12 / 21(57)$ & 0.493 \\
\hline
\end{tabular}


Table 1 (continued)

\begin{tabular}{lll}
\hline Parameter & $\begin{array}{l}\text { Control group, } \\
n=75\end{array}$ & $\begin{array}{l}\text { APT group, } \\
n=85\end{array}$ \\
\hline Deep & $0 / 20(0)$ & $1 / 21(5)$ \\
Combined & $6 / 20(30)$ & $8 / 21(38)$ \\
Laterality of DCI-related infarction, $n(\%)$ & & \\
Unilateral & $13 / 20(65)$ & $14 / 21(66)$ \\
Right side & $10 / 20$ & $8 / 21$ \\
Left side & $3 / 20$ & $6 / 21$ \\
Bilateral & $7 / 20(35)$ & $7 / 21(33)$ \\
Lesion volume $\left(\mathrm{cm}^{3}\right)$, median $(\mathrm{IQR})$ & $78.5(24.7-282.6)$ & $33.7(6.1-107.5)$ \\
\hline
\end{tabular}

Statistically significant differences are made italics $(P<0.05)$

$A T P$ antiplatelet therapy, IQR interquartile range, WFNS World Federation of Neurosurgical Societies, CSF cerebrospinal fluid, ICA internal carotid artery, $P C O m A$ posterior communicating artery, $A C o m A$ anterior communicating artery, $A C A$ anterior cerebral artery, $M C A$ middle cerebral artery, $W E B$ Woven EndoBridge; $V P$ ventriculoperitoneal, $D C I$ delayed cerebral ischemia, $C V S$ cerebral vasospasm, $F V$ flow velocity, $T C D$ transcranial Doppler, $D I N D$ delayed ischemic neurologic deficit, $\mathrm{PBrO}_{2}$ brain tissue oxygenation, $\mathrm{PCT}$ perfusion computed tomography

${ }^{a}$ Basilar artery and AComA aneurysms were excluded

$n=32,38 \%$ ) were (temporary) analgosedated and mechanically ventilated. Sixty-one patients (38\%) (control group $n=$ 34, 45\%; APT group $n=27,32 \%$ ) required long-term analgosedation due to poor-grade aSAH (WFNS IV-V) or complications in the acute clinical course and received invasive neuromonitoring including $\mathrm{PBrO}_{2}$ which was placed bilaterally in the majority of patients $(44 / 61,72 \%)$. Twenty-nine patients showed functional deterioration with a decrease of $\mathrm{PBrO}_{2}$ (control group 19/34, 56\%; APT group 10/27, 37\%; $P=0.198)$. PCT examinations were performed in 106 patients (control group 53/75, 71\%; APT group 53/85, 62\%). DCI-related hypoperfusion was observed significantly more often in the control group (34/53, 64\%) compared with the APT group (18/53, $34 \% ; P=0.003)$. Routine TCD measurements showed a significant increase in $\mathrm{FV}$ in about $1 / 3$ of the patients (control group $n=25,33 \%$; APT group $n=30,35 \%$; $P=0.868$ ).

Angiographic CVS was present in $41 \%$ of patients in either group $(P=1.000$; Table 2$)$. The further analysis of characteristic angiographic CVS pattern showed a similar distribution of the investigated parameters between the control and APT group. In particular, there were no differences between the groups with regard to the number of affected vessels and the severity of angiographic CVS. Moreover, a similar number of patients received ERT, mostly IAN applications (Table 2). Of the 51 patients treated with stent-assisted coiling, 19 patients developed symptomatic angiographic CVS (37\%). In the majority of cases $(n=15 / 19,79 \%)$, the stented vessel segment was not or only mildly affected by CVS. In case of unilateral occurrence, CVS was primary detected ipsilateral to the ruptured aneurysm $(n=24 / 27,88 \%)$.

In the multivariable analysis of factors associated with the occurrence of angiographic CVS (Table 3), poor WFNS grade independently increased the risk for angiographic CVS in our cohort $(P=0.026, \mathrm{OR}=2.15,95 \%$ CI $1.10-4.23)$, while male sex showed a non-significant statistical trend toward a reduced risk for angiographic CVS $(P=0.055, \mathrm{OR}=0.48,95 \%$ CI 0.23 1.02). The use of APT had no significant impact on the occurrence of angiographic CVS.

Regarding the incidence of DCI-related infarctions in our collective, we found no significant differences between the study groups (27\% in the control group vs. $25 \%$ in the APT group, $P=0.875$; Table 1). A detailed analysis of the infarct characteristics showed comparable patterns with respect to the number of vascular territories involved, the location or the laterality (Table 1). Although the median lesion volume did not differ significantly between the groups, we found a statistical trend toward higher absolute values in the control group $78.5 \mathrm{~cm}^{3}$ (IQR 24.7282.6) vs $33.7 \mathrm{~cm}^{3}$ (IQR 6.1-107.5) in the APT group $(P=$ 0.090). The lesion overlay plots of patients with DCI-related infarctions are shown in Fig. 1. It visually appeared that the patients in the control group developed more often larger areas of infarction, especially in the left hemisphere (Fig. 1a). In the lesion overlap image of the two subgroups of APT patients (aspirin mono APT vs. DAPT), it visually appeared that patients with DAPT had especially small lesion overlaps (Fig. 1b). This impression could also be statistically supported, because the median lesion volume was significantly smaller in the DAPT subgroup (14.3 $\mathrm{cm}^{3}$ (IQR 5.1-57.8) than in the control group (78.5 $\mathrm{cm}^{3}$ (IQR 24.7-282.6), $P=0.011$ ).

Multivariable analyses (Table 3 ) showed that a poor WFNS grade at admission was the only independent predictor of DCI-related infarction in our cohort $(P=0.001$, OR $=3.63$, 95\% CI 1.73-7.61).

Functional outcome after 3 months is shown in Fig. 2. The rate of favorable (mRS $\leq 2)$ outcome $(62 \%$ in the APT group vs. $41 \%$ in the control group, $P=0.011$; Fig. 2a) was significantly higher in the APT group than in controls. Comparing the outcome of patients who were admitted with WFNS grade I-III aSAH, functional outcome was 
Table 2 Parameters regarding angiographic CVS patterns in the study cohort

\begin{tabular}{|c|c|c|c|}
\hline Parameter & Control group $(n=75), n(\%)$ & APT group $(n=85), n(\%)$ & $P$ value \\
\hline Occurrence of angiographic CVS & $31(41)$ & $35(41)$ & 1.000 \\
\hline \multicolumn{4}{|l|}{ No. of affected vessels per patient } \\
\hline 1 vessel & $2 / 31(6)$ & $4 / 35(11)$ & \multirow[t]{3}{*}{0.709} \\
\hline $2-3$ vessels & $12 / 31(39)$ & $13 / 35(37)$ & \\
\hline$>3$ vessels & $17 / 31(55)$ & $16 / 35(46)$ & \\
\hline \multicolumn{4}{|c|}{ Affected vessel segment per patient } \\
\hline Proximal & $30 / 31(98)$ & $30 / 35(86)$ & \multirow[t]{2}{*}{0.784} \\
\hline Distal & $23 / 31(74)$ & $23 / 35(66)$ & \\
\hline \multicolumn{4}{|l|}{ Lateralization of CVS } \\
\hline Unilateral & $17 / 31(55)$ & $21 / 35(60)$ & \multirow[t]{2}{*}{0.804} \\
\hline Bilateral & $14 / 31(45)$ & $14 / 35(40)$ & \\
\hline \multicolumn{4}{|l|}{ Localization of CVS } \\
\hline Anterior circulation & $31 / 31(100)$ & $34 / 35(97)$ & \multirow[t]{2}{*}{0.403} \\
\hline Posterior circulation & $10 / 31(31)$ & $8 / 35(23)$ & \\
\hline \multicolumn{4}{|l|}{ Severity of CVS } \\
\hline Mild to moderate & $8 / 31(26)$ & $13 / 35(37)$ & \multirow[t]{2}{*}{0.429} \\
\hline Severe & $23 / 31(74)$ & $22 / 35(63)$ & \\
\hline Endovascular rescue therapy & $31(41)$ & $35(41)$ & \\
\hline IAN & $30 / 31(98)$ & $32 / 35(91)$ & 0.615 \\
\hline TBA & $12 / 31(39)$ & $9 / 35(26)$ & 0.426 \\
\hline
\end{tabular}

$A P T$ antiplatelet therapy, CVS cerebral vasospasm, IAN intra-arterial nimodipine treatment, TBA transarterial balloon angioplasty significantly better in the APT group (favorable outcome in $83 \%$ in the APT vs. $57 \%$ in the control group, $P=0.004$ : Fig. 2 b), while only a small proportion of poor-grade patients (WFNS grade IV-
V) in both groups showed favorable functional outcome after 3 months $(12 \%$ in the APT vs. $17 \%$ in the control group, $P=$ 0.708: Fig. 2c).

Table 3 Logistic regression analysis of factors associated with the occurrence of angiographic CVS or DCI-related infarction

\begin{tabular}{|c|c|c|c|c|c|c|c|c|}
\hline \multirow{3}{*}{ Parameter } & \multicolumn{4}{|l|}{ Angiographic CVS } & \multicolumn{4}{|c|}{ DCI-related infarction } \\
\hline & \multicolumn{2}{|c|}{ Univariable analysis } & \multicolumn{2}{|c|}{ Multivariable analysis } & \multicolumn{2}{|c|}{ Univariable analysis } & \multicolumn{2}{|c|}{ Multivariable analysis } \\
\hline & OR [CI 0.95] & $P$ value & OR [CI 0.95] & $P$ value & OR [CI 0.95] & $P$ value & OR [CI 0.95] & $P$ value \\
\hline Older age ( $>55$ years) & 0.85 [0.45-1.59] & 0.610 & - & - & 1.88 [0.91-3.88] & 0.087 & - & - \\
\hline Male sex & $0.48[0.23-1.00]$ & 0.049 & $0.48[0.23-1.02]$ & 0.055 & $0.54[0.23-1.28]$ & 0.159 & - & - \\
\hline WFNS grade $4-5$ & $2.16[1.11-4.22]$ & 0.023 & $2.15[1.10-4.23]$ & 0.026 & $3.63[1.73-7.6]$ & $<0.001$ & $3.63[1.73-7.61]$ & 0.001 \\
\hline Fisher scale 3 & $0.77[0.41-1.46]$ & 0.426 & - & - & $0.87[0.43-1.78]$ & 0.706 & - & - \\
\hline Occurrence of IVH & $1.41[0.74-2.67]$ & 0.298 & - & - & $2.69[1.30-5.56]$ & 0.008 & $1.56[0.65-3.73]$ & 0.317 \\
\hline $\begin{array}{l}\text { Aneurysm localization } \\
\text { Posterior circulation }\end{array}$ & $0.55[0.26-1.16]$ & 0.117 & - & - & $0.40[0.15-1.02]$ & 0.056 & - & - \\
\hline Antiplatelet therapy & 0.99 [0.53-1.87] & 0.984 & - & - & $0.90[0.44-1.84]$ & 0.777 & - & - \\
\hline
\end{tabular}

Data comparisons were made using univariable and multivariable analysis. Statistically significant differences are made italics $(P<0.05)$

CVS cerebral vasospasm, DCI delayed cerebral ischemia, OR Odds ratio, $C I$ confidence interval, WFNS World Federation of Neurosurgical Societies, IVH intraventricular hemorrhage 
Fig. 1 Lesion overlay plots showing DCI-related cerebral infarctions in the control and the APT group (a) and, in a further subgroup analysis, infarct lesions inpatients receiving aspirin monotherapy or dual APT (aspirin plus clopidogrel) (b). The color bar indicates the number of overlapping lesions (minimum $n=2$ )
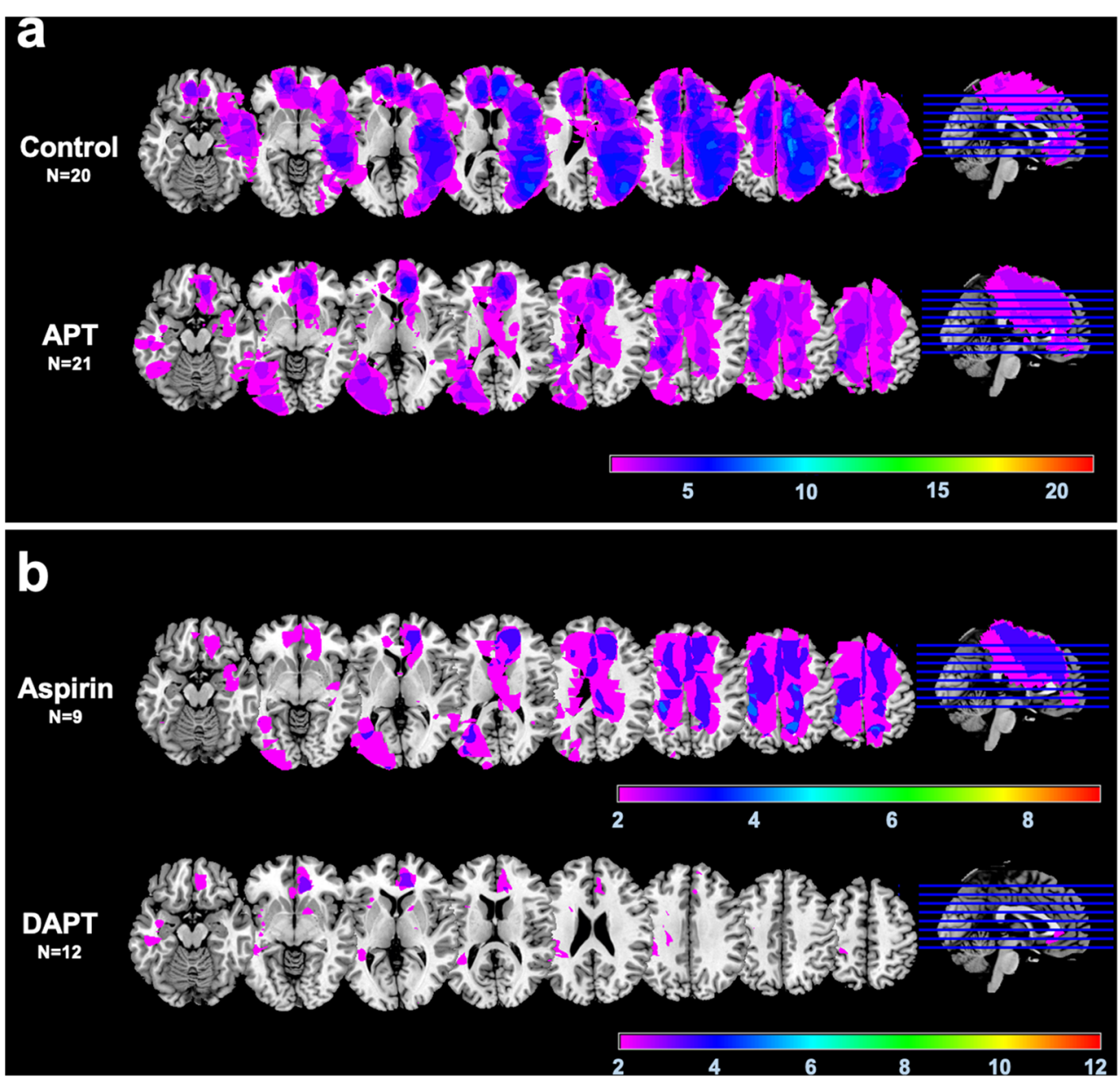

After adjustment for potential confounders, multivariable analysis confirmed an independent association between APT and a lower rate of unfavorable functional outcome after 3 months ( $P=0.021, \mathrm{OR}=0.40,95 \%$ CI $0.19-0.87$; Table 4$)$. In contrast, the factors "older age ( $>55$ years)" $(P=0.014, \mathrm{OR}=2.67,95 \%$ CI 1.22-5.86) and "poor WFNS grade (IV-V)" $(P<0.001$, $\mathrm{OR}=16.23,95 \%$ CI $6.49-40.58)$ increased the risk for an unfavorable functional outcome (Table 4).

Due to the significant smaller volume of DCI-related infarction in patients with DAPT, we performed a further subgroup analysis within the treatment group. When splitting the APT group into "aspirin monotherapy" and "DAPT," univariable subgroup analysis showed that DAPT was significantly associated with a lower rate of unfavorable outcome at 3 months $(P=0.021$, OR $=0.43,95 \%$ CI $0.21-0.88)$ while aspirin monotherapy still showed a statistical trend $(P=$ $0.068, \mathrm{OR}=0.44,95 \%$ CI $0.18-1.06)$.

\section{Discussion}

Post-interventional APT was found to be an independent predictor of a better functional outcome after 3 months in the
aSAH patients of our cohort. These results are in line with previous studies reporting on improved outcome in aSAH patients who received antiplatelets after aneurysm treatment $[4,11,14]$. Although the exact mode of action by which antiplatelets influence outcome after aSAH is not yet clarified, it has been previously suggested that platelet inhibitors reduce the risk of DCI and thereby have a beneficial effect on clinical outcome. Accordingly, several studies investigated a possible preventive effect of different antiplatelet agents on the development of DCI, but those trials are quite heterogeneous and thus difficult to interpret. A former systematic review of clinical studies, investigating microsurgically treated patients only, concluded that APT reduces the risk of DCI resulting in an increased rate of good outcome after aSAH [14]. However, only three of the five included trials provided data on the occurrence of DCI and only one of them used aspirin as trial medication. Noteworthy, this small, randomized trial found an equal distribution of clinical and radiologic DCI in both groups [20].

A more recent Cochrane review demonstrated a nonsignificant trend toward a lower incidence of DCI and a better functional outcome among patients treated with antiplatelets after aSAH [15]. But again, the validity of this meta-analysis 
Fig. 2 Stacked bar charts of overall functional outcome after 3 months in the control and the APT group (a) as well as in WFNS grade I-III (b) and WFNS IV-V (c) patients compared between the control and the APT group. The bars indicate the proportions of patients with favorable (mRS 0-2) and unfavorable outcome (mRS 3-6) with further subdivision into death (mRS 6) and loss of follow-up (LOF)
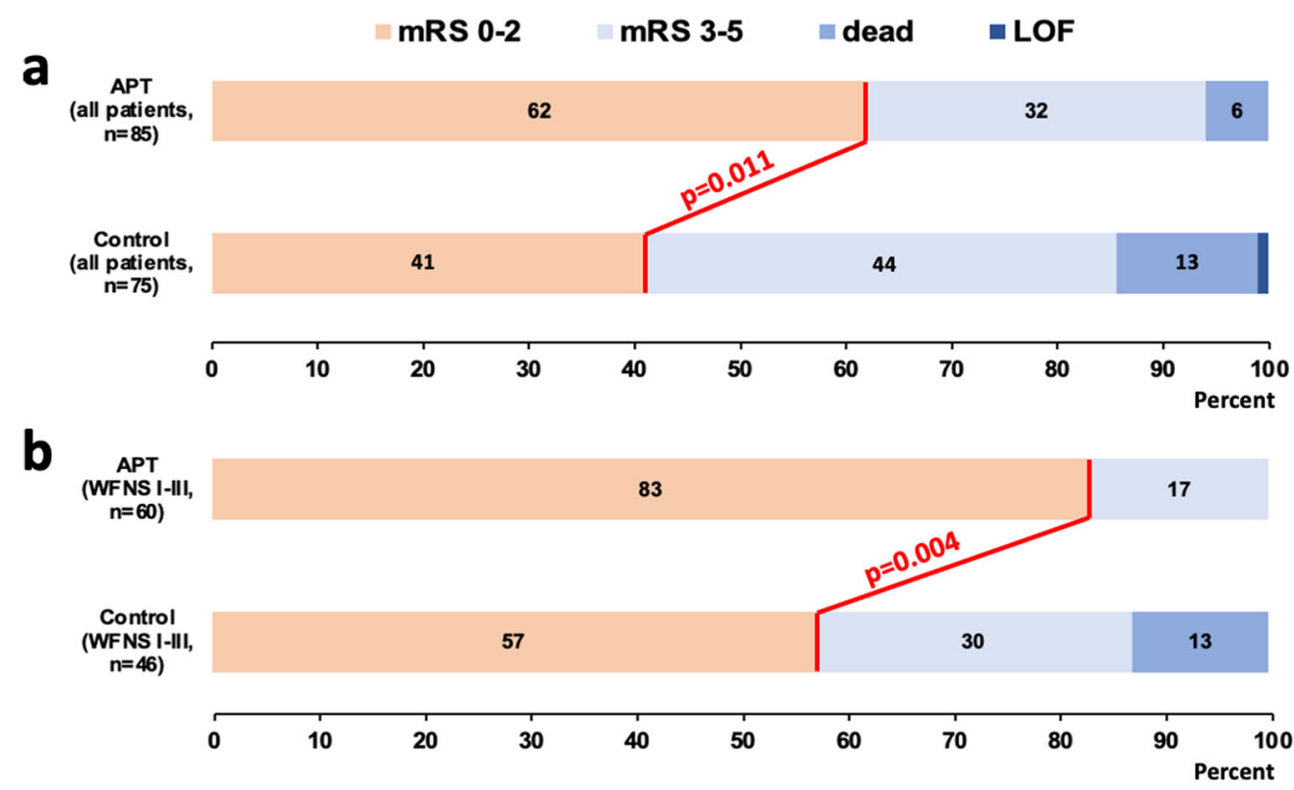

C

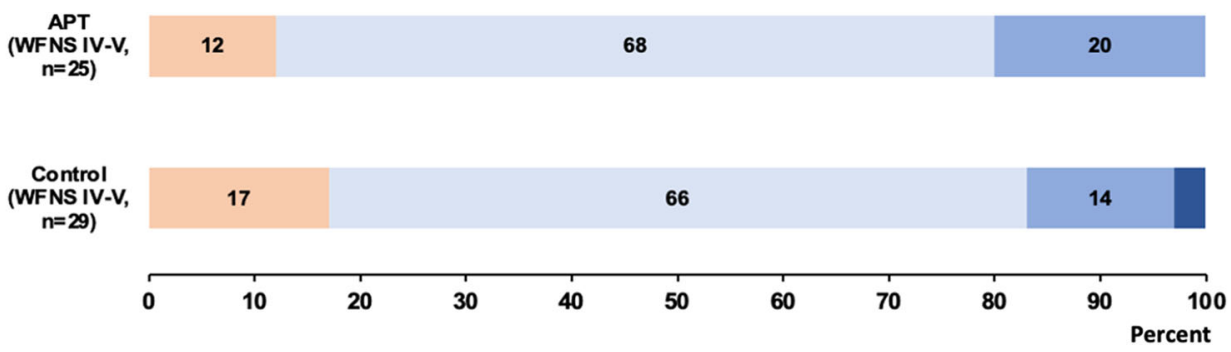

is limited due to the differences of the studies included such as the trial medication used or the start of antiplatelet treatment. Moreover, the majority of these patients were treated by neurosurgical clipping, so that the results are hardly transferable to endovascularly treated aSAH patients, who constitute the group of patients that usually needs APT.
In our cohort of endovascularly treated patients, we found no significant difference with regard to the overall occurrence of clinical DCI and DCI-related infarction between patients receiving APT and those without. Likewise, the occurrence of angiographic CVS was comparable in both groups. These results seem surprising at first, especially in view of the better
Table 4 Logistic regression analysis of factors associated with unfavorable functional outcome $(\mathrm{mRS} \geq 3)$

\begin{tabular}{|c|c|c|c|c|}
\hline \multirow[b]{2}{*}{ Parameter } & \multicolumn{2}{|c|}{ Univariable analysis } & \multicolumn{2}{|l|}{ Multivariable analysis } \\
\hline & OR [CI 0.95] & $\begin{array}{l}P \\
\text { value }\end{array}$ & OR [CI 0.95] & $P$ value \\
\hline Older age ( $>55$ years) & $2.09[1.11-3.93]$ & 0.023 & $2.67[1.22-5.86]$ & 0.014 \\
\hline Male sex & $0.80[0.40-1-61]$ & 0.534 & - & - \\
\hline WFNS grade 4-5 & $\begin{array}{l}14.25 \\
\quad[6.01-33.76]\end{array}$ & $<0.001$ & $16.23[6.49-40.58]$ & $<0.001$ \\
\hline Fisher scale 3 & $0.66[0.35-1.24]$ & 0.193 & - & - \\
\hline Occurrence of IVH & $4.80[2.43-9.50]$ & $<0.001$ & 1.99 [0.81-4.89] & 0.132 \\
\hline Aneurysm localization posterior circulation & $1.17[0.58-2.36]$ & 0.669 & - & - \\
\hline Antiplatelet therapy & $0.44[0.23-0.82]$ & 0.010 & $0.40[0.19-0.87]$ & 0.021 \\
\hline
\end{tabular}

Data comparisons were made using univariable and multivariable analysis. Statistically significant differences are made italics $(P<0.05)$

$m R S$ modified Rankin scale, $O R$ odds ratio, $C I$ confidence interval, WFNS World Federation of Neurosurgical Societies, $I V H$ intraventricular hemorrhage 
functional outcome of the APT group in our study. But there are several indications in the literature that improved outcome through antiplatelets cannot be equated with the prevention of DCI. A randomized controlled trial assessing whether aspirin administration reduces the risk of DCI in SAH patients failed to demonstrate such an effect and concluded that aspirin does not reduce the occurrence of DCI. Still, functional outcome tended to be better in patients who received aspirin, especially after endovascular aneurysm treatment [45]. Similarly, the largest and most recent meta-analysis to date analyzing the use of antiplatelet drugs among 2822 patients with aSAH found significantly reduced rates of poor outcome and mortality among the APT group, but antiplatelet use did not significantly decrease DCI or CVS rates [4]. In this study, the majority of patients $(75 \%)$ were treated endovascularly and $95 \%$ of the patients received aspirin as post-interventional APT. Interestingly, this meta-analysis found a trend toward lower DCI among the subgroup of endovascularly treated patients. Similar positive effects of APT on the occurrence DCI were reported by two recent series of endovascularly treated aSAH patients [11,29]. The results of our study, however, do not confirm these findings in endovascularly treated aSAH patients as we could not demonstrate any differences in the overall occurrence of DCI and angiographic CVS in our collective. There are indications in the literature that the reduction of DCI after antiplatelet administration tended to be lower in patients with a high Fisher score (Fisher 3-4) [4]. As the amount of cisternal blood represents an independent risk factor for CVS and DCI [6, 7, 27], it seems reasonable that the various pathophysiological mechanisms that contribute to the development of CVS and DCI might be enhanced by the severity of the initial bleeding event. In this case, the beneficial effects of antiplatelets might be less pronounced or the chosen dosage might be too low. As the vast majority of patients in our cohort presented with Fisher grade 3-4, this condition might have influenced our results.

Due to the discrepancy between improved outcome and absence of overall CVS and DCI reduction in patients with postinterventional APT, we performed a detailed analysis of the severity and the distribution patterns of angiographic CVS and of DCIrelated infarction. Interestingly, we found no significant differences between the groups with regard to the severity, the vessels or vascular territories affected, or any other investigated parameter. The analysis of the infarct lesion volumes demonstrated a nonsignificant statistical trend toward a higher lesion volume in the control group, and the descriptive lesion analyses in our study indicated that the patients in the control group developed more often larger infarctions with a predominance in the left hemisphere. Moreover, the results of the subgroup analysis suggest that especially the DAPT treatment might have a protective effect. The beneficial effect of APT on functional outcome in our cohort might therefore be mediated by the reduction of the lesion size, especially when DAPT was administered which was the case in the majority
(2/3) of our patients receiving antiplatelet medication. In this context, it is interesting that PCT examinations in the APT group demonstrated DCI-related cerebral hypoperfusion significantly less often compared with the control group.

Previous studies proposed that the positive impact of APT on clinical outcome might be associated with a decreased occurrence of microthrombi $[4,45]$. Several clinical and autopsy studies reported that the formation of microthrombi and the associated impairment of cerebral microcirculation contributes to the development of DCI and cerebral infarction after SAH [34, 41, 42, 49]. An autopsy study showed that the amount of microemboli is significantly increased in SAH patients with large areas of DCI-related cerebral ischemia [42]. APT might therefore reduce the formation or the amount of microemboli and thus the extension of DCI-related cerebral infarction in aSAH patients. While aspirin irreversibly inhibits the enzyme cyclooxygenase (COX) and thus the subsequent biosynthesis of prostaglandins and thromboxane, clopidogrel blocks platelet aggregation through the $\mathrm{P}_{2} \mathrm{Y}_{12}$-receptor pathway [16]. These mechanisms lead to synergistic effects with regard to the inhibition of platelet aggregation. The combination of the two drugs has been shown to be more effective than aspirin alone in reducing the risk of ischemic events in patients with acute coronary syndromes [2] as well as the risk of recurrent stroke in the acute phase after an ischemic event in neurological patients [23,32]. Our study provides indications that such an additive beneficial effect from DAPT could also apply for DCI-related infarctions in aSAH patients.

So far, data about potential benefits and risks associated with DAPT after aSAH are scarce and inconsistent. A recent study of patients presenting with Hunt and Hess grade I-III showed a significantly reduced risk for clinical CVS and DCI but could not demonstrate a positive effect of DAPT on functional outcome [29]. A second retrospective study of 329 aSAH patients receiving either aspirin $(92.4 \%)$ or DAPT (7.4\%) demonstrated decreased DCI rates and improved functional outcome in the entire APT group compared with the control group. However, a subgroup analysis of the DAPT cohort showed no significant impact on the occurrence of DCI. Moreover, patients with DAPT had higher in-hospital mortality, lower favorable outcome, and an increased risk of major bleeding events [11]. A second study, comparing the outcomes among aSAH patients who received aspirin monotherapy vs. DAPT, also failed to demonstrate a superior beneficial effect of DAPT reporting a trend toward higher bleeding complication rates in the DAPT group as well $[11,51]$.

In view of these findings, the potential risks of DAPT in neurosurgical patients must be discussed. Several studies show increased rates of intracranial bleeding complications due to single-antiplatelet use in aSAH patients [14, 15, 44]. With regard to DAPT, two recent studies by Hudson et al. demonstrate a higher risk for radiographic hemorrhage associated with external ventricular drain or VP shunt placement 
in aSAH patients requiring DAPT due to stent-assisted coiling or flow diversion. However, the risk of clinically significant bleeding complications was considered low [21, 22]. A recent meta-analysis [5] reported that ventriculostomy-related bleeding rates were significantly higher among patients receiving APT and that the rate of major hemorrhage was higher after DAPT compared with single APT. But the overall rate of major hemorrhage was also low in this study. In our study, we found no significant differences in the rate of hemorrhagic complications between the investigated groups. Major bleeding events occurred only in a small number of patients. However, all of those individuals received DAPT, and the size of our study groups was probably too small to detect statistically significant differences of major bleeding events in patients with DAPT. Our results in general confirm the assumption that clinically relevant bleeding events are rare in aSAH patients with APT, but are tending to occur more often in DAPT than in mono APT.

Our results confirm that a poor WFNS grade (IV-V) is a strong predictor of DCI-related infarction and unfavorable outcome after aSAH. Interestingly, our clinical outcome analysis revealed that only APT patients presenting with WFNS grade I-III showed a better functional outcome, whereas the outcome of patients with WFNS grade IV-V was similar between both groups. Our results suggest that postinterventional APT fails to improve functional outcome in WFNS grade IV-V patients, at least in our study cohort. Poor-grade aSAH patients are not only characterized by a particularly severe early brain injury (EBI), but also by the risk of developing severe medical complications during the acute clinical course. The extent of platelet activation and inflammation has been shown to be related to the severity of EBI after aSAH [19]. One could therefore speculate that the antiplatelet effect or the mode of action of the drugs used was insufficient in these severely affected patients or that the dosage might not have been high enough. In this context, a major limitation of our study must be discussed. The individual responder status to APT has not been routinely analyzed especially not in patients receiving aspirin monotherapy. This issue is at the same time a major shortcoming of previous studies dealing with APT after aSAH as even randomized and prospective studies have not acknowledged APT responder status $[15,45]$. It is well known that the individual responsiveness to antiplatelet drugs varies among patients and is influenced by genetic polymorphism but also by clinical factors such as platelet hyperactivity, drug-drug interactions, and poor adsorption [1, 43]. The reported rates of aspirin and clopidogrel range widely in the literature [8], but it has been shown that impaired responsiveness to aspirin is common in acute brain ischemia and is also associated with worse neurological deficits at stroke onset, early clinical deterioration, and poorer functional outcome [40]. In analogy to these findings, impaired antiplatelet responsiveness might also be associated with the severity of EBI in poor-grade SAH patients suggesting that there might have been an overestimation of APTresponders in our study. Given the possibility that an early inhibition of platelet function during EBI might be crucial with regard to the further course of the disease, poor WFNS grade patients in particular might benefit from a higher aspirin dosage or the additional administration of clopidogrel. In this context, DAPT with an early loading dose of aspirin and clopidogrel might be beneficial, and our study confirmed a sufficient response to both drugs in the majority of patients. Future prospective studies should acknowledge this important aspect.

Our study is moreover limited by its retrospective design and the small cohort size affecting the clinical significance of our results. As we excluded patients who received surgical aneurysm treatment, our results are not transferable to all aSAH patients. However, patients who underwent aneurysm clipping are not well suited as a control group, as craniotomy, cisternal blood removal, or microsurgical manipulation of the brain and its vasculature might have an incalculable impact on the study results. As the investigated groups in our study were defined according to the need of post-interventional APT, another limitation is given by a certain selection bias with regard to the type of endovascular aneurysm treatment as a relevant number of patients in the APT group received stent-assisted coiling. The decision on what type of endovascular therapy was used might have been influenced not only by the complexity of the aneurysm configuration but also by the clinical factors or the individual assessment of the interventional neuroradiologist. The use of stent-assisted coiling may carry some beneficial effects regarding the functional outcome in the APT group, as patients who received coiling only without post-interventional APT might be at higher risk for ischemic cerebral events.

Compared with previous studies, we used DCI-related infarction as an independent outcome measure in multivariable analysis instead of overall clinical and/or radiological DCI. Due to the heterogeneity of the study cohort consisting of awake and analgosedated aSAH patients, data on clinical DCI were only partially available limiting the power of statistical analysis. However, we assume that the occurrence of DCI-related cerebral infarction has a greater impact on functional outcome than temporary neurological deterioration associated with DIND. Moreover, a strict clinical definition of DCI in previous studies often resulted in an underrepresentation of poor-grade $\mathrm{SAH}$ patients, who at the same time are at the highest risk for ischemic complications [39].

Finally, reliable data on previous antiplatelet longterm medication or one-time antiplatelet intake in connection with the acute headache event were not available for all patients and therefore could not be considered in the analysis. Thus, previous antiplatelet medication before admission might also have influenced the results of this study. 


\section{Conclusion}

Post-interventional APT was associated with a lower rate of unfavorable outcome in our aSAH cohort without an increased risk for intracranial bleeding events. The overall incidence of angiographic CVS or DCI-related infarction was not reduced in the APT group. However, with regard to the volume of DCI-related infarctions, our results suggest that APT, especially DAPT, may reduce the lesion's size and thereby improves clinical outcome. Our results underline the need for prospective randomized trials to assess and compare potential benefits and risks of monoand DAPT in patients with aSAH and to answer the clinical question of whether the use of APT is indicated in patients after aSAH in order to improve clinical outcome.

Authors' contribution All the authors contributed to the study conception and design. Acquisition of data, analysis, and interpretation were performed by Claudia Ditz, Björn Machner, Hannes Schacht, and Alexander Neumann. Statistical analyses were performed by Claudia Ditz and Jan Küchler. The first draft of the manuscript was written by Claudia Ditz and Jan Küchler, and all the authors commented on previous versions of the manuscript. All the authors read and approved the final manuscript and gave their consent for publication.

Funding Open Access funding enabled and organized by Projekt DEAL.

Data availability The data that support the findings of this study are available from the corresponding author, upon reasonable request.

\section{Compliance with ethical standards}

Conflict of interest The authors declare that they have no conflicts of interest.

Ethics approval This article does not contain any studies with human participants or animals performed by any of the authors.

Consent to participate For this type of retrospective study formal consent is not required.

Consent for publication Not applicable.

Code availability Not applicable.

Open Access This article is licensed under a Creative Commons Attribution 4.0 International License, which permits use, sharing, adaptation, distribution and reproduction in any medium or format, as long as you give appropriate credit to the original author(s) and the source, provide a link to the Creative Commons licence, and indicate if changes were made. The images or other third party material in this article are included in the article's Creative Commons licence, unless indicated otherwise in a credit line to the material. If material is not included in the article's Creative Commons licence and your intended use is not permitted by statutory regulation or exceeds the permitted use, you will need to obtain permission directly from the copyright holder. To view a copy of this licence, visit http://creativecommons.org/licenses/by/4.0/.

\section{References}

1. Angiolillo DJ, Bernardo E, Sabate M, Jimenez-Quevedo P, Costa MA, Palazuelos J, Hernandez-Antolin R, Moreno R, Escaned J, Alfonso F, Banuelos C, Guzman LA, Bass TA, Macaya C, Fernandez-Ortiz A (2007) Impact of platelet reactivity on cardiovascular outcomes in patients with type 2 diabetes mellitus and coronary artery disease. J Am Coll Cardiol 50:1541-1547. https:// doi.org/10.1016/j.jacc.2007.05.049

2. Bowry AD, Brookhart MA, Choudhry NK (2008) Meta-analysis of the efficacy and safety of clopidogrel plus aspirin as compared to antiplatelet monotherapy for the prevention of vascular events. Am J Cardiol 101:960-966. https://doi.org/10.1016/j.amjcard.2007.11. 057

3. Budohoski KP, Guilfoyle M, Helmy A, Huuskonen T, Czosnyka M, Kirollos R, Menon DK, Pickard JD, Kirkpatrick PJ (2014) The pathophysiology and treatment of delayed cerebral ischaemia following subarachnoid haemorrhage. J Neurol Neurosurg Psychiatry 85:1343-1353. https://doi.org/10.1136/jnnp-2014-307711

4. Cagnazzo F, Derraz I, Lefevre PH, Gascou G, Dargazanli C, Riquelme C, Perrini P, di Carlo D, Bonafe A, Costalat V (2019) Antiplatelet therapy in patients with aneurysmal SAH: impact on delayed cerebral ischemia and clinical outcome. A meta-analysis. AJNR Am J Neuroradiol 40:1201-1206. https://doi.org/10.3174/ ajnr.A6086

5. Cagnazzo F, Di Carlo DT, Petrella G, Perrini P (2020) Ventriculostomy-related hemorrhage in patients on antiplatelet therapy for endovascular treatment of acutely ruptured intracranial aneurysms. A meta-analysis. Neurosurg Rev 43:397-406. https:// doi.org/10.1007/s10143-018-0999-0

6. Charpentier C, Audibert G, Guillemin F, Civit T, Ducrocq X, Bracard S, Hepner H, Picard L, Laxenaire MC (1999) Multivariate analysis of predictors of cerebral vasospasm occurrence after aneurysmal subarachnoid hemorrhage. Stroke 30: 1402-1408. https://doi.org/10.1161/01.str.30.7.1402

7. Claassen J, Bernardini GL, Kreiter K, Bates J, Du YE, Copeland D, Connolly ES, Mayer SA (2001) Effect of cisternal and ventricular blood on risk of delayed cerebral ischemia after subarachnoid hemorrhage: the Fisher scale revisited. Stroke 32:2012-2020. https:// doi.org/10.1161/hs0901.095677

8. Collet JP, Montalescot G (2009) Platelet function testing and implications for clinical practice. J Cardiovasc Pharmacol Ther 14: 157-169. https://doi.org/10.1177/1074248409339309

9. Connolly ES Jr, Rabinstein AA, Carhuapoma JR, Derdeyn CP, Dion J, Higashida RT, Hoh BL, Kirkness CJ, Naidech AM, Ogilvy CS, Patel AB, Thompson BG, Vespa P, American Heart Association Stroke C, Council on Cardiovascular R, Intervention, Council on Cardiovascular N, Council on Cardiovascular S, Anesthesia, Council on Clinical C (2012) Guidelines for the management of aneurysmal subarachnoid hemorrhage: a guideline for healthcare professionals from the American Heart Association/ american Stroke Association. Stroke 43:1711-1737. https://doi. org/10.1161/STR.0b013e3182587839

10. Crowley RW, Medel R, Dumont AS, Ilodigwe D, Kassell NF, Mayer SA, Ruefenacht D, Schmiedek P, Weidauer S, Pasqualin A, Macdonald RL (2011) Angiographic vasospasm is strongly correlated with cerebral infarction after subarachnoid hemorrhage. Stroke 42:919-923. https://doi.org/10.1161/STROKEAHA.110. 597005

11. Darkwah Oppong M, Gembruch O, Pierscianek D, Kohrmann M, Kleinschnitz C, Deuschl C, Monninghoff C, Kaier K, Forsting M, Sure U, Jabbarli R (2019) Post-treatment antiplatelet therapy reduces risk for delayed cerebral ischemia due to aneurysmal subarachnoid hemorrhage. Neurosurgery 85:827-833. https://doi.org/ 10.1093/neuros/nyy550 
12. Ditz C, Hartlieb M, Neumann A, Machner B, Schacht H, Krajewski KL, Leppert J, Tronnier VM, Kuchler J (2020) Routine use of perfusion computed tomography for the detection of delayed cerebral ischemia in unconscious patients after aneurysmal subarachnoid hemorrhage. Acta Neurochir 163:151-160. https://doi.org/10. 1007/s00701-020-04571-8

13. Dolatowski K, Malinova V, Frolich AM, Schramm R, Haberland U, Klotz E, Mielke D, Knauth M, Schramm P (2014) Volume perfusion CT (VPCT) for the differential diagnosis of patients with suspected cerebral vasospasm: qualitative and quantitative analysis of 3D parameter maps. Eur J Radiol 83:1881-1889. https://doi.org/ 10.1016/j.ejrad.2014.06.020

14. Dorhout Mees SM, Rinkel GJ, Hop JW, Algra A, van Gijn J (2003) Antiplatelet therapy in aneurysmal subarachnoid hemorrhage: a systematic review. Stroke 34:2285-2289. https://doi.org/10.1161/ 01.STR.0000083621.44269.3E

15. Dorhout Mees SM, van den Bergh WM, Algra A, Rinkel GJ (2007) Antiplatelet therapy for aneurysmal subarachnoid haemorrhage. Cochrane Database Syst Rev CD006184. https://doi.org/10.1002/ 14651858.CD006184.pub2

16. Dorsam RT, Kunapuli SP (2004) Central role of the P2Y12 receptor in platelet activation. J Clin Invest 113:340-345. https://doi.org/10. 1172/JCI20986

17. Dorsch NW (1995) Cerebral arterial spasm-a clinical review. Br J Neurosurg 9:403-412. https://doi.org/10.1080/ 02688699550041403

18. Frontera JA, Fernandez A, Schmidt JM, Claassen J, Wartenberg KE, Badjatia N, Connolly ES, Mayer SA (2009) Defining vasospasm after subarachnoid hemorrhage: what is the most clinically relevant definition? Stroke 40:1963-1968. https://doi.org/10.1161/ STROKEAHA.108.544700

19. Frontera JA, Provencio JJ, Sehba FA, McIntyre TM, Nowacki AS, Gordon E, Weimer JM, Aledort L (2017) The role of platelet activation and inflammation in early brain injury following subarachnoid hemorrhage. Neurocrit Care 26:48-57. https://doi.org/10. 1007/s12028-016-0292-4

20. Hop JW, Rinkel GJ, Algra A, Berkelbach van der Sprenkel JW, van Gijn J (2000) Randomized pilot trial of postoperative aspirin in subarachnoid hemorrhage. Neurology 54:872-878. https://doi.org/ 10.1212/wnl.54.4.872

21. Hudson JS, Nagahama Y, Nakagawa D, Starke RM, Dlouhy BJ, Torner JC, Jabbour P, Allan L, Derdeyn CP, Greenlee JDW, Hasan D (2018) Hemorrhage associated with ventriculoperitoneal shunt placement in aneurysmal subarachnoid hemorrhage patients on a regimen of dual antiplatelet therapy: a retrospective analysis. J Neurosurg 129:916-921. https://doi.org/10.3171/2017.5. JNS17642

22. Hudson JS, Prout BS, Nagahama Y, Nakagawa D, Guerrero WR, Zanaty M, Chalouhi N, Jabbour P, Dandapat S, Allan L, OrtegaGutierrez S, Samaniego EA, Hasan D (2019) External ventricular drain and hemorrhage in aneurysmal subarachnoid hemorrhage patients on dual antiplatelet therapy: a retrospective cohort study. Neurosurgery 84:479-484. https://doi.org/10.1093/neuros/nyy 127

23. Johnston SC, Easton JD, Farrant M, Barsan W, Conwit RA, Elm JJ, Kim AS, Lindblad AS, Palesch YY, Clinical Research Collaboration NETTN, the PI (2018) Clopidogrel and aspirin in acute ischemic stroke and high-risk TIA. N Engl J Med 379:215225. https://doi.org/10.1056/NEJMoa1800410

24. Juvela S (1995) Aspirin and delayed cerebral ischemia after aneurysmal subarachnoid hemorrhage. J Neurosurg 82:945-952. https:// doi.org/10.3171/jns.1995.82.6.0945

25. Juvela S, Hillbom M, Kaste M (1991) Platelet thromboxane release and delayed cerebral ischemia in patients with subarachnoid hemorrhage. J Neurosurg 74:386-392. https://doi.org/10.3171/jns. 1991.74.3.0386
26. Juvela S, Ohman J, Servo A, Heiskanen O, Kaste M (1991) Angiographic vasospasm and release of platelet thromboxane after subarachnoid hemorrhage. Stroke 22:451-455. https://doi.org/10. 1161/01.str.22.4.451

27. Lee H, Perry JJ, English SW, Alkherayf F, Joseph J, Nobile S, Zhou LL, Lesiuk H, Moulton R, Agbi C, Sinclair J, Dowlatshahi D (2018) Clinical prediction of delayed cerebral ischemia in aneurysmal subarachnoid hemorrhage. J Neurosurg:1-8. https://doi.org/10. 3171/2018.1.JNS172715

28. Leng LZ, Fink ME, Iadecola C (2011) Spreading depolarization: a possible new culprit in the delayed cerebral ischemia of subarachnoid hemorrhage. Arch Neurol 68:31-36. https://doi.org/10.1001/ archneurol.2010.226

29. Nagahama Y, Allan L, Nakagawa D, Zanaty M, Starke RM, Chalouhi N, Jabbour P, Brown RD, Derdeyn CP, Leira EC, Broderick J, Chimowitz M, Torner JC, Hasan D (2018) Dual antiplatelet therapy in aneurysmal subarachnoid hemorrhage: association with reduced risk of clinical vasospasm and delayed cerebral ischemia. J Neurosurg 129:702-710. https://doi.org/10.3171/2017. 5.JNS17831

30. Ohkuma H, Ogane K, Fujita S, Manabe H, Suzuki S (1993) Impairment of anti-platelet-aggregating activity of endothelial cells after experimental subarachnoid hemorrhage. Stroke 24:15411545; discussion 1545-1546. https://doi.org/10.1161/01.str.24.10. 1541

31. Ohkuma H, Suzuki S, Kimura M, Sobata E (1991) Role of platelet function in symptomatic cerebral vasospasm following aneurysmal subarachnoid hemorrhage. Stroke 22:854-859. https://doi.org/10. 1161/01.str.22.7.854

32. Prasad K, Siemieniuk R, Hao Q, Guyatt G, O'Donnell M, Lytvyn L, Heen AF, Agoritsas T, Vandvik PO, Gorthi SP, Fisch L, Jusufovic M, Muller J, Booth B, Horton E, Fraiz A, Siemieniuk J, Fobuzi AC, Katragunta N, Rochwerg B (2018) Dual antiplatelet therapy with aspirin and clopidogrel for acute high risk transient ischaemic attack and minor ischaemic stroke: a clinical practice guideline. BMJ 363: k5130. https://doi.org/10.1136/bmj.k5130

33. Rabinstein AA, Weigand S, Atkinson JL, Wijdicks EF (2005) Patterns of cerebral infarction in aneurysmal subarachnoid hemorrhage. Stroke 36:992-997. https://doi.org/10.1161/01.STR. $0000163090.59350 .5 \mathrm{a}$

34. Romano JG, Forteza AM, Concha M, Koch S, Heros RC, Morcos JJ, Babikian VL (2002) Detection of microemboli by transcranial Doppler ultrasonography in aneurysmal subarachnoid hemorrhage. Neurosurgery 50:1026-1030; discussion 1030-1021. https://doi. org/10.1097/00006123-200205000-00016

35. Roos YB, de Haan RJ, Beenen LF, Groen RJ, Albrecht KW, Vermeulen M (2000) Complications and outcome in patients with aneurysmal subarachnoid haemorrhage: a prospective hospital based cohort study in the Netherlands. J Neurol Neurosurg Psychiatry 68:337-341. https://doi.org/10.1136/jnnp.68.3.337

36. Rorden C, Karnath HO, Bonilha L (2007) Improving lesionsymptom mapping. J Cogn Neurosci 19:1081-1088. https://doi. org/10.1162/jocn.2007.19.7.1081

37. Rosengart AJ, Schultheiss KE, Tolentino J, Macdonald RL (2007) Prognostic factors for outcome in patients with aneurysmal subarachnoid hemorrhage. Stroke 38:2315-2321. https://doi.org/10. 1161/STROKEAHA.107.484360

38. Rowland MJ, Hadjipavlou G, Kelly M, Westbrook J, Pattinson KT (2012) Delayed cerebral ischaemia after subarachnoid haemorrhage: looking beyond vasospasm. Br J Anaesth 109:315-329. https://doi.org/10.1093/bja/aes264

39. Sarrafzadeh AS, Vajkoczy P, Bijlenga P, Schaller K (2014) Monitoring in neurointensive care - the challenge to detect delayed cerebral ischemia in high-grade aneurysmal SAH. Front Neurol 5: 134. https://doi.org/10.3389/fneur.2014.00134 
40. Schwammenthal Y, Tsabari R, Shenkman B, Schwartz R, Matetzky S, Lubetsky A, Orion D, Israeli-Korn S, Chapman J, Savion N, Varon D, Tanne D (2008) Aspirin responsiveness in acute brain ischaemia: association with stroke severity and clinical outcome. Cerebrovasc Dis 25:355-361. https://doi.org/10.1159/000118382

41. Stein SC, Browne KD, Chen XH, Smith DH, Graham DI (2006) Thromboembolism and delayed cerebral ischemia after subarachnoid hemorrhage: an autopsy study. Neurosurgery 59:781-787; discussion 787-788. https://doi.org/10.1227/01.NEU.0000227519. 27569.45

42. Suzuki S, Kimura M, Souma M, Ohkima H, Shimizu T, Iwabuchi T (1990) Cerebral microthrombosis in symptomatic cerebral vasospasm-a quantitative histological study in autopsy cases. Neurol Med Chir (Tokyo) 30:309-316. https://doi.org/10.2176/ nmc.30.309

43. Topcuoglu MA, Arsava EM, Ay H (2011) Antiplatelet resistance in stroke. Expert Rev Neurother 11:251-263. https://doi.org/10.1586/ ern.10.203

44. Toussaint LG 3rd, Friedman JA, Wijdicks EF, Piepgras DG, Pichelmann MA, McIver JI, McClelland RL, Nichols DA, Meyer FB, Atkinson JL (2004) Influence of aspirin on outcome following aneurysmal subarachnoid hemorrhage. J Neurosurg 101:921-925. https://doi.org/10.3171/jns.2004.101.6.0921

45. van den Bergh WM, Group MS, Algra A, Dorhout Mees SM, van Kooten F, Dirven CM, van Gijn J, Vermeulen M, Rinkel GJ (2006) Randomized controlled trial of acetylsalicylic acid in aneurysmal subarachnoid hemorrhage: the MASH study. Stroke 37:23262330. https://doi.org/10.1161/01.STR.0000236841.16055.0f

46. van den Bergh WM, Kerr RS, Algra A, Rinkel GJ, Molyneux AJ, International Subarachnoid Aneurysm Trial Collaborative G (2009) Effect of antiplatelet therapy for endovascular coiling in aneurysmal subarachnoid hemorrhage. Stroke 40:1969-1972. https://doi.org/ 10.1161/STROKEAHA.108.528802

47. van Gijn J, Kerr RS, Rinkel GJ (2007) Subarachnoid haemorrhage. Lancet 369:306-318. https://doi.org/10.1016/S0140-6736(07) 60153-6

48. Vanhoutte PM, Houston DS (1985) Platelets, endothelium, and vasospasm. Circulation 72:728-734. https://doi.org/10.1161/01. cir.72.4.728

49. Vergouwen MD, Vermeulen M, Coert BA, Stroes ES, Roos YB (2008) Microthrombosis after aneurysmal subarachnoid hemorrhage: an additional explanation for delayed cerebral ischemia. $\mathrm{J}$ Cereb Blood Flow Metab 28:1761-1770. https://doi.org/10.1038/ jcbfm. 2008.74

50. Vergouwen MD, Vermeulen M, van Gijn J, Rinkel GJ, Wijdicks EF, Muizelaar JP, Mendelow AD, Juvela S, Yonas H, Terbrugge KG, Macdonald RL, Diringer MN, Broderick JP, Dreier JP, Roos YB (2010) Definition of delayed cerebral ischemia after aneurysmal subarachnoid hemorrhage as an outcome event in clinical trials and observational studies: proposal of a multidisciplinary research group. Stroke 41:2391-2395. https://doi.org/10.1161/ STROKEAHA.110.589275

51. Wallace AN, Kayan Y, Almandoz JED, Mulder M, Milner AA, Scholz JM, Stiernagle K, Contestabile E, Tipps ME (2020) Dual antiplatelet therapy does not improve outcomes after aneurysmal subarachnoid hemorrhage compared with aspirin monotherapy. Clin Neurol Neurosurg 195:106038. https://doi.org/10.1016/j. clineuro.2020.106038

Publisher's note Springer Nature remains neutral with regard to jurisdictional claims in published maps and institutional affiliations. 